\title{
Spare Parts Surgery-Salvage of a Below Knee Amputation Stump
}

\author{
Chasari Tancharoen, Edmund Ek*, Anand Ramakrishnan
}

Department of Plastics Reconstructive Surgery, Royal Melbourne Hospital, Melbourne, Australia.

Email: *edmund_ek@hotmail.com

Received January $14^{\text {th }}, 2012$; revised February $19^{\text {th }}$, 2012; accepted March 23 ${ }^{\text {rd }}, 2012$

\begin{abstract}
"Spare parts surgery" is well-described in the plastic surgery literature. In the setting of trauma, otherwise discarded parts can be utilized for reconstruction resulting in a superior functional and aesthetic outcome for the patient. We describe the use of spare part surgery in order to preserve a functional knee articulation in a patient undergoing amputation for compartment.
\end{abstract}

Keywords: Below Knee Amputation; Free Flap; Fillet of Sole Flap; Spare Part Surgery

\section{Introduction}

Lower limb amputation is commonly required for trauma, ischaemia, infection and tumour. Below knee amputation (BKA) is considered superior to above knee amputation (AKA) with regards to both metabolic requirements of ambulation and aesthetics. The requirements of a BKA stump include a functional knee joint, adequate tibial length and a well-padded, sensate, sturdy stump able to fit a prosthesis and withstand stresses of ambulation. When these requirements cannot be met, AKA is usually the only option. Use of a composite flap harvested from the otherwise discarded part is well accepted. We present use of a free sole of foot flap raised from the amputated limb to provide adequate wound coverage. This type of "spare parts" surgery resulted in the maximal functional and aesthetic outcome possible for our patient.

\section{Case Report}

A 34 year-old male intravenous drug user (IVDU) presented to our hospital with acute compartment syndrome of the right lower limb. This followed an unspecified period of unconsciousness during which the patient's limb was crushed beneath a television set. At presentation the patient was alert but disorientated and in severe pain. He was afebrile, tachycardic and hypotensive. His right lower limb was severely swollen with tense, tight compartments, paralysis and severe pain with passive movement. There was altered sensation in the sural, tibial and common peroneal nerve distributions. Dorsalis pedis and posterior tibial pulsations were present. Radiographs demon-

${ }^{*}$ Corresponding author. strated no fracture. The patient was in acute renal failure with a creatinine of $259 \mathrm{umol} / \mathrm{L}$ and creatinine kinase of $149863 \mathrm{IU} / \mathrm{L}$.

The patient was resuscitated and taken to theatre for decompression. Thigh fasciotomy was performed through a longitudinal incision over the lateral compartment. Thigh muscles were seen to be viable, bleeding and reactive to stimulation. Leg fasciotomies were performed by standard two-incision technique. At the time all muscles were noted to be discoloured and minimally reactive to stimulation. Foot fasciotomies were not performed. Minimal debridement was performed and wounds were dressed with vacuum assisted closure $\left(\mathrm{VAC}^{\odot}\right)$ dressings and the patient transferred to the intensive care unit for further management.

Thirty-six hours after initial surgery, the patient was returned to theatre for debridement. At this operation all thigh muscles were viable while only the medial gastrocnemius muscle was viable in the leg. Over the following nine days the patient was taken to theatre three times for serial debridement of necrotic muscles with preservation of anterior tibial, posterior tibial and peroneal neurovascular bundles. Given the severity of the injury and lack of useful leg motors, the decision was made to proceed with amputation.

At the time of amputation it was found that the remnant medial head of gastrocnemius would not provide sufficient coverage for a below knee stump. To avoid AKA, while providing a well-padded and stable stump, the sole of foot was harvested as a free flap. The posterior tibial neurovascular bundle was isolated posterior to the medial malleolus with release of the flexor retinaculum and dissection into the sole of the foot. The skin/muscle 
flap was then raised to include the heel pad and extended to the metatarsal heads. The plane of dissection was superficial to the interosseous muscles (Figure 1). The tourniquet was subsequently released to demonstrate perfusion of the flap prior to division of the vessels and nerve. Amputation was then performed and the flap inset with the heel pad overlying the tibial stump. Vascular anastomosis was performed end-to-end to the posterior tibial vessels using $8 / 0$ monofilament nylon and the tibial nerve coapted to the proximal tibial nerve using $8 / 0$ monofilament.

Postoperatively the patient had an uneventful recovery with transfer to rehabilitation at day 14 (Figure 2). At 6 months the flap is well healed with improving sensation to the skin flap and satisfactory weight-bearing within a prosthesis (Figure 3).

\section{Discussion}

Lower limb amputation comes at significant cost to both the patient and to the community. For patients, the psychological, emotional and physical costs of limb amputation are significant. They experience physical pain and emotional stress while recovering from surgery and during the rehabilitation phase. They face significant alteration of their lifestyle, loss of physical capabilities, the

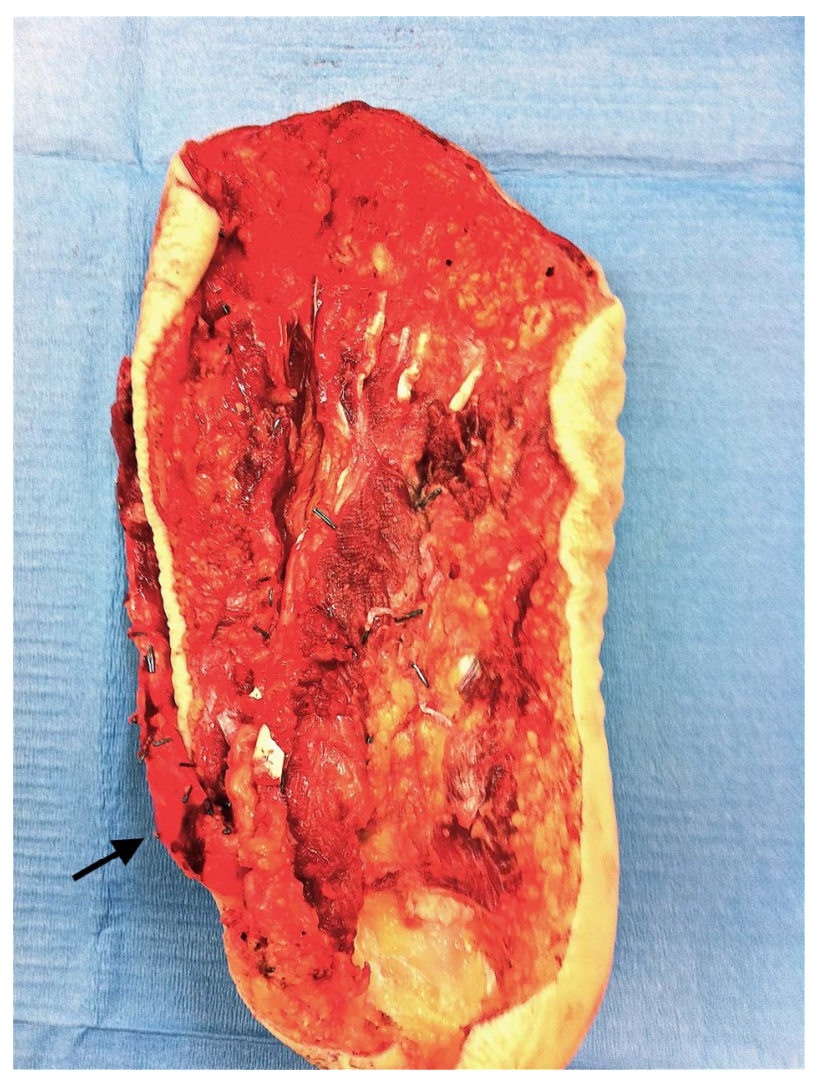

Figure 1. Fillet of sole free flap (arrow-posterior tibial neurovascular bundle).

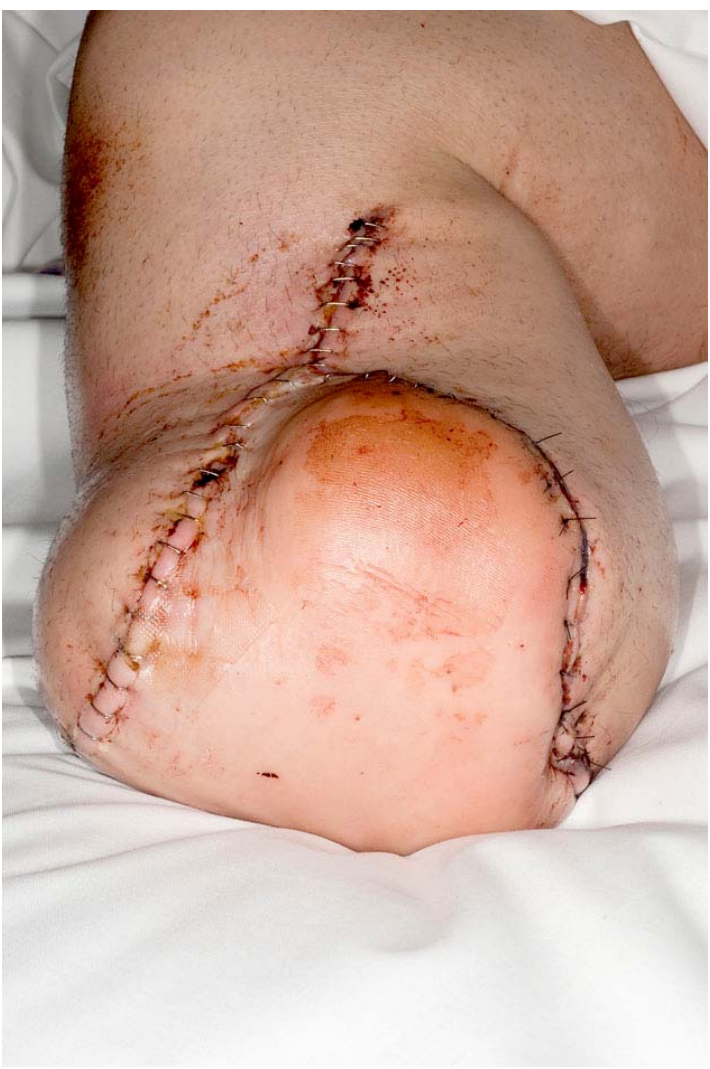

Figure 2. Day 7 post-operative.

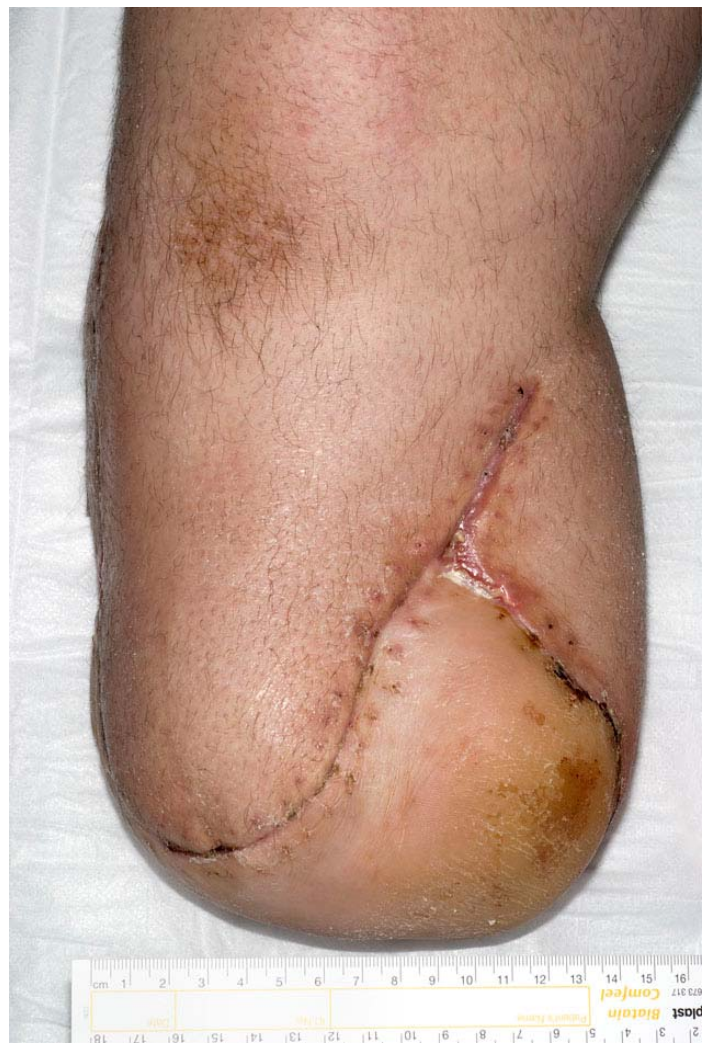

Figure 3. 4 Weeks post-operative. 
need for occupational re-training and potential difficultties with social reintegration [1]. Socioeconomically, limb amputation has been demonstrated to be more expensive than limb salvage [2]. Consequently, amputation remains the choice of last resort.

Amputation level has significant impact on patient rehabilitation with BKA well recognized as being functionally and aesthetically superior to AKA. Functionally, unilateral amputation, distal amputation level and younger age has been shown to be predictive of better walking ability [3]. Comparison of metabolic requirements of walking within a prosthesis also demonstrates energy expenditure of trans-tibial amputees to be the lowest with trans-femoral amputees the highest [4]. Hence, when possible, BKA remains the amputation level of choice. This requires a functional knee articulation, sufficient tibial length and adequate soft tissue coverage to provide a wellpadded, robust and ideally sensate stump able to withstand the rigours of ambulating within a prosthesis. If the soft tissue requirements cannot be met then further tibial shortening, through-knee amputation, AKA or free tissue transfer become the only remaining options.

"Spare part" surgery is well described in the literature [5-8]. In the setting of lower limb amputation with inadequate soft tissue coverage use of tissue from the amputated segment allows preservation of functional length of the stump without additional donor site morbidity. Numerous fillet flaps have been described with the fillet sole flap considered ideal for resurfacing of a BKA stump. By definition, fillet flaps are axial pattern flaps that can function as composite tissue transfers for reconstruction of complex defects [9]. The fillet sole flap can be easily raised on the posterior tibial neurovascular pedicle and provides adequate size, soft tissue bulk, stability and strength to withstand the shear forces associated with walking. In our patient a dorsalis pedis flap or perforator-based fasciocutaneous flap could have also been used for re-surfacing but these would have lacked the bulk and strength of the heel pad/sole of foot. Use of the "spare part" provides single stage reconstruction, relative ease of flap dissection and no further donor site morbidity in a patient already undergoing major surgery. With a two-team approach, operative time can be further shortened with harvesting of the flap performed on a side table after amputation of the limb.

While this flap could have been raised as a pedicled flap, preserving continuity of the tibial nerve, this would introduce the difficulties of lengthy dissection within an inflamed field, pedicle redundancy and risk of kinking during flap inset.

\section{Conclusion}

The advantages of this type of surgery are obvious. Be- sides the avoidance of donor site morbidity, the other goals of BKA are met giving patients maximal functional and aesthetic results. In this case, the availability of the entire sole and heel pad provided an ideal salvage optionsoft tissue bulk, thick durable skin, ability to withstand sheer forces [2]. Tibial nerve coaptation also provides the potential for sensory reinnervation. While this option is limited in patients suffering peripheral vascular disease, it remains a superior option to AKA in patients requiring amputation for trauma, tumour and infection [3].

\section{REFERENCES}

[1] M. Akula, S. Gella, C. J. Shaw, P. McShane and A. M. Mohsen, "A Meta-Analysis of Amputation versus Limb Salvage in Mangled Lower Limb Injuries-The Patient Perspective,” Injury, Vol. 42, No. 11, 2011, pp. 1194-1197. doi:10.1016/j.injury.2010.05.003

[2] K. C. Chung, D. Saddawi-Konefka, S. C. Haase and G. Kaul, "A Cost-Utility Analysis of Amputation Versus Salvage for Gustilo Type IIIB and IIIC Open Tibial Fractures,” Plastic and Reconstructive Surgery, Vol. 124, No. 6, 2009, pp. 1965-1973. doi:10.1097/PRS.0b013e3181bcf156

[3] K. Sansam, V. Neumann, R. O’Connor and B. Bhakta, "Predicting Walking Ability Following Lower Limb Amputation: A Systematic Review of the Literature,” Journal of Rehabilitation Medicine, Vol. 41, No. 8, 2009, pp. 593603. doi:10.2340/16501977-0393

[4] A. S. Goktepe, B. Cakir, B. Yilmaz and K. Yazicioglu, "Energy Expenditure of Walking with Prostheses: Comparison of Three Amputation Levels," Prosthetics and Orthotics International, Vol. 34, No. 1, 2010, pp. 31-36. doi:10.3109/03093640903433928

[5] G. J. Gumley, A. M. MacLeod, S. Thistlethwaite and A. R. Ryan, "Case Report: Total Cutaneous Harvesting from an Amputated Foot-Two Free Flaps Used for Acute Reconstruction,” British Journal of Plastic Surgery, Vol. 40, No. 3, 1987, pp. 313-316. doi:10.1016/0007-1226(87)90131-7

[6] Y. C. Chiang, F. C. Wei, J. W. Wang and W. S. Chen, "Reconstruction of Below-Knee Stump Using the Salvaged Foot Fillet Flap,” Plastic and Reconstructive Surgery, Vol. 96, No. 3, 1995, pp. 731-738. doi:10.1097/00006534-199509000-00031

[7] W. A. Morrison, B. O’Brien and A. M. MacLeod, "The Foot as a Donor Site in Reconstructive Microsurgery," World Journal of Surgery, Vol. 3, No. 1, 1979, pp. 43-52. doi:10.1007/BF01556386

[8] J. P. Ver Halen, P. Yu, R. J. Skoracki and D. W. Chang, "Reconstruction of Massive Oncologic Defects Using Free Fillet Flaps," Plastic and Reconstructive Surgery, Vol. 125, No. 3, 2010, pp. 913-922. doi:10.1097/PRS.0b013e3181cb6548

[9] M. V. Kuntscher, D. Erdmann, H. H. Homann, H. U. Steinau, S. L. Levin and G. Germann, "The Concept of Fillet Flaps: Classification, Indications, and Analysis of Their Clinical Value," Plastic and Reconstructive Surgery, Vol. 108, No. 4, 2001, pp. 885-896. doi:10.1097/00006534-200109150-00011 